\title{
Chest CT Results in Diagnosis of Post-traumatic Haemato Pneumatocele
} Adriana Nohemi Garcia Herrera*

Pre-hospital Emergency Medical Services (UVI-Movil Pamplona), Pamplona, Spain
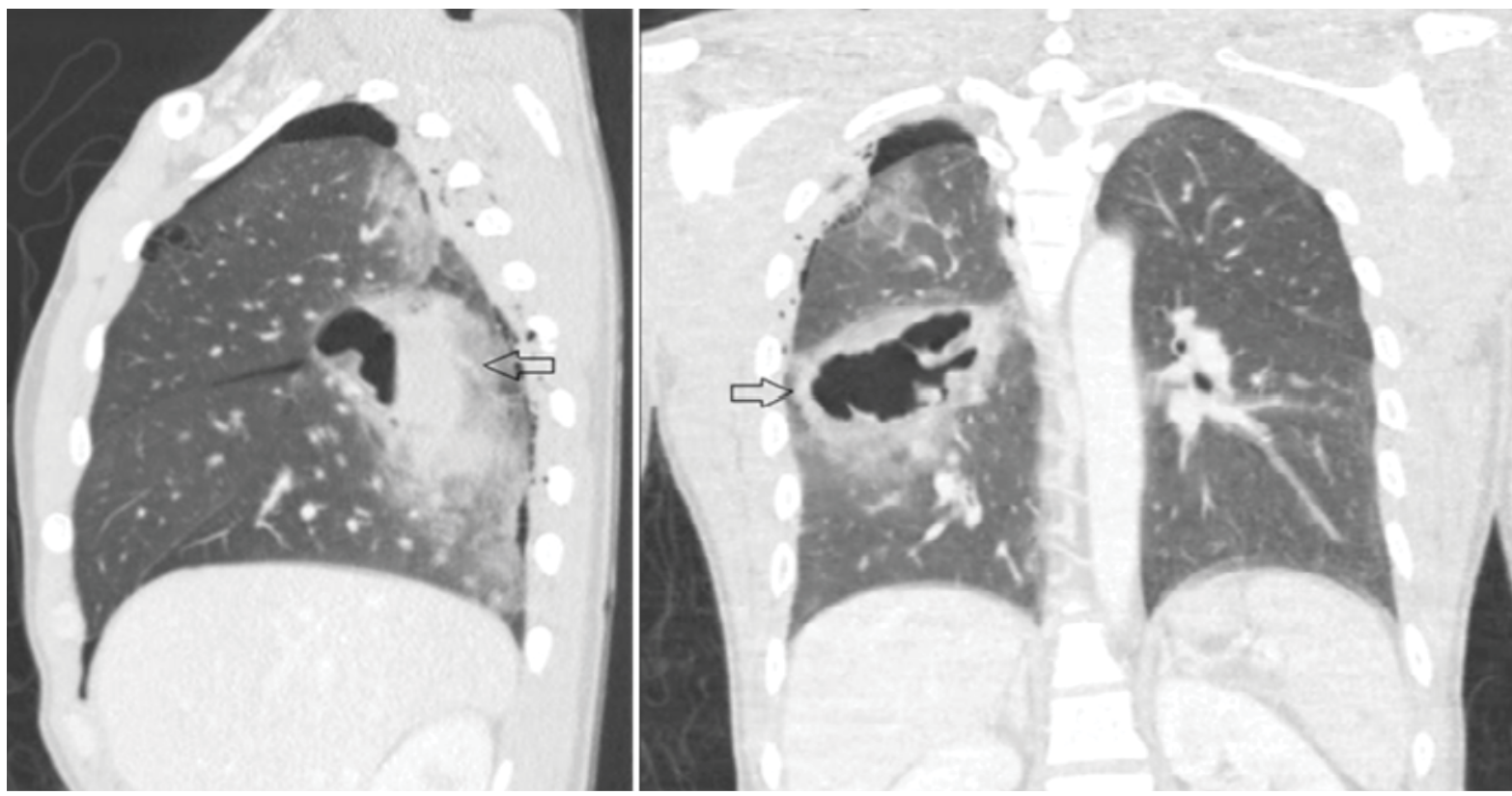

Figure 1: Chest CT (sagittal and coronal planes), right haemato-pneumatocele (arrow).

\section{Clinical Image}

A 37-year-old man presented to the Emergency department with multiple injuries after he was hit by a van. He was conscious, hemodynamically and respiratory stable. CT image showed a right-sided round collection suggestive of haemato-pneumatocele (arrows), small pneumomediastinum and hemoneumothorax as well as right rib fractures $\left(3^{\text {rd }}\right.$ through $\left.7^{\text {th }}\right)$ (Figure 1$)$. Haematopneumatocele is an air/blood cavitary lesion in the lung parenchyma following blunt thoracic trauma. It is characterized by minor clinical symptoms and major radiologic signs. The formation of this lesion requires a direct violent impact which explains its frequency in young adults. Though rarely described, its frequency is certainly underestimated. Their clinical implications are minimal and have a favorable evolution with spontaneous resolution of the radiological abnormalities. No aggressive intervention is required. This patient had an uncomplicated clinical course.

*Corresponding author: Adriana Nohemi Garcia Herrera, Plaza Sancho Abarca 1, 3 B 31014, Pamplona, Navarra, Spain, Tel: +351 229410 100; E-mail: herrera.adriana@gmail.com

Citation: Herrera ANG (2017) Chest CT Results in Diagnosis of Post-traumatic Haemato Pneumatocele. Int J Clin Med Imaging 4: 581. doi:10.4172/23760249.1000581

Copyright: ( $) 2017$ Herrera ANG. This is an open-access article distributed under the terms of the Creative Commons Attribution License, which permits unrestricted use, distribution, and reproduction in any medium, provided the original author and source are credited. 